\title{
Caracterização geofísica da região norte de Minas Gerais (Jaíba, Montes Claros e Bocaiúva).
}

Teixeira, C. D. ${ }^{1}$; Von Huelsen, M. G. ${ }^{1}$; Chimpliganond, C. N. ${ }^{1}$; Lima, J. S. ${ }^{1}$; Barros, L. V. ${ }^{1}$

1 - Universidade de Brasília - IGD/ObSis

Copyright 2014, SBGf - Sociedade Brasileira de Geofísica

Este texto foi preparado para a apresentação no VI Simpósio Brasileiro de Geofísica, Porto Alegre, 14 a 16 de outubro de 2014. Seu conteúdo foi revisado pelo Comitê Técnico do VI SimBGf, mas não necessariamente representa a opinião da SBGf ou de seus associados. É proibida a reprodução total ou parcial deste material para propósitos comerciais sem prévia autorização da SBGt.

\section{Abstract}

This work presents the geophysical characterization of regions Jaíba, Montes Claros and Bocaiúva (Minas Gerais) focusing on processing and interpretation of gammaspectrometric data. That was accomplished by analysing the false color ternary composition (RGB) where the relation between the three radioelements $\mathrm{K}$, eU and $\mathrm{eTh}$ and delimit regions with similar gammaspectometric signature is noticed. It is also presented Magnetometric data by analyzing the grid of the Amplitude of Analytic Signal (ASA), featuring domains and magnetic lineaments and later a possible relationship of these lineaments with the seismicity of the region of Montes Claros region.

\section{Introdução}

A sismicidade na região de Montes Claros (Minas Gerais), não é um fenômeno recente. O Observatório Sismológico da Universidade de Brasília registrou no período de 1995 a maio/2012 vinte e dois tremores, sendo o maior de magnitude 4,2mR ocorrido em 19/05/2012. Entretanto, o recente evento de maior magnitude impulsionou um estudo mais detalhado dessa atividade em trabalho. Apesar da intensificação dos estudos na região de Montes Claros, devido à sismicidade dessa região ter se tornado um fenômeno frequente, até hoje muitas informações sobre a área continuam desconhecidas. Dentro desse contexto, procurou-se realizar um trabalho que pudesse fornecer informações para uma maior compreensão do arcabouço geológico e geofísico da região de Montes Claros. Este trabalho contempla dados de sismologia oriundos de uma rede Sismológica conjunta entre UnB e USP e também os dados aerogeofísicos (gamaespectrometria e magnetometria) cedidos pela Companhia de Desenvolvimento Econômico de Minas Gerais (CODEMIG), dados estes referentes à área $11 \mathrm{~A}$ abrangida pelos municípios de Jaíba, Montes Claros e Bocaiúva.

\section{Contexto Geológico}

A região está inserida principalmente no Cráton São Francisco e uma pequena porção na Faixa de Dobramentos Araçuaí (Figura 1).
O cráton por sua vez é definido por Almeida (1977), é uma unidade geotectônica que ocupa grande parte do território mineiro, apresentando-se delimitado pela Faixa Brasília (a oeste) e pelo Orógeno Araçuaí (a sul e a leste). Almeida (1977) considera que o final do processo de cratonização deu-se durante o Transamazônico.

A geologia da região é caracterizada por sedimentos das formações Três Marias e Lagoa do Jacaré, pertencentes ao Grupo Bambuí, formações Serra do Catuni e Duas Barras, pertencentes ao Grupo Macaúbas, estes pertencentes ao Supergrupo São Francisco, de idade neoproterozóica, além das formações Galho do Miguel e Sopa Brumadinho, pertencentes ao Grupo Diamantina, estes pertencentes ao Supergrupo Espinhaço. Dentro dos domínios de bacia sedimentar existe o predomínio de rochas sedimentares com ocorrência de metamórficas. As rochas sedimentares são representadas, em sua grande maioria, pelos calcários pré-cambriano do grupo Bambuí. A litologia presente no complexo do Espinhaço apresenta 0 predomínio de rochas metamórficas e ígneas, grande ocorrência de metamorfismo regional, áreas de maciço antigo e escarpamentos orientados por fraturas. Existe o predomínio de quartzito, filitos e rochas plutônicas. (CPRM, 2003). Além dessas formações também ocorrem coberturas Colúvio-Aluviais e Eluviais cenozóicas que recobrem extensas áreas de Minas Gerais.

\section{Localização da Área}

Geologicamente a área está inserida principalmente no Cráton São Francisco e uma pequena porção na Faixa de Dobramentos Araçuaí (Figura 1). A área estende-se desde o norte do estado até a porção central (Figura 1a) e onde engloba as Folhas Cartográficas (SD-23-Z) (SE23-X) (SE-23-Z), na escala de 1:500.000.

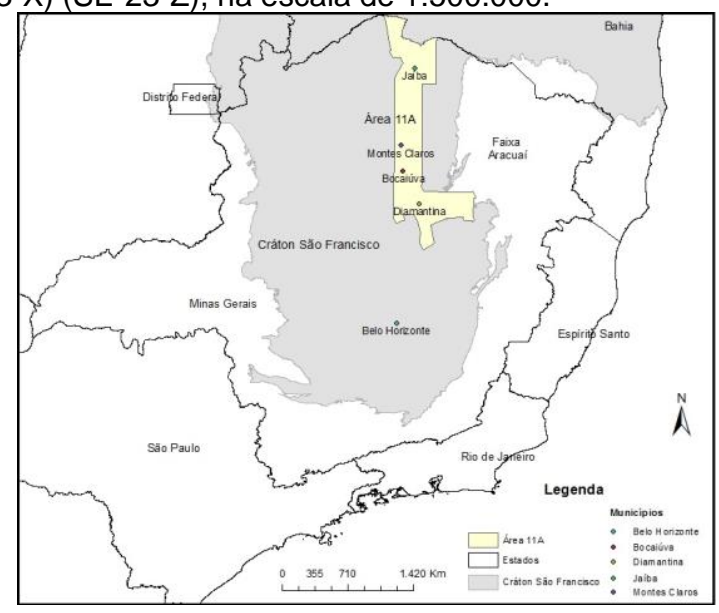

Figura 1 - Mapa de localização da área. 


\section{Metodologia/ Problema Investigado}

Os dados geofísicos utilizados no presente trabalho são de gamaespectometria e magnetometria do Programa de Levantamento Aerogeofísico 2008/2009, dados estes cedidos pela Companhia de Desenvolvimento Econômico de Minas Gerais (CODEMIG). Esses dados de alta resolução foram obtidos com altura média de voo de 100 $\mathrm{m}$, sendo a direção das linhas de voo para $25^{\circ} \mathrm{NW}$ e espaçamento de $0,5 \mathrm{~km}$, e a direção das linhas de controle $65^{\circ} \mathrm{NE}$ com espaçamento de $10 \mathrm{~km}$.

O processamento dos dados foi feito com a utilização do software Oasis montaj da Geosoft versão 8.0. As malhas foram geradas utilizando o método de interpolação de curvatura mínima com tamanho das células de 1/4 da distância entre as linhas de voo, 125m.

Foram obtidos mapas gamaespectrométricos correspondentes aos canais de $\mathrm{K}$, Th, $\mathrm{U}$ e de contagem total. Da combinação dos três canais ( $K, \mathrm{U}$ e Th) foram obtidas as composições ternárias RGB (red, green, blue) e CMY (cyan, magenta, yellow). Em seguida foi elaborado um modelo digital de terreno (MDT), que posterior foi utilizado como uma quarta componente nas combinações RGB e CMY.

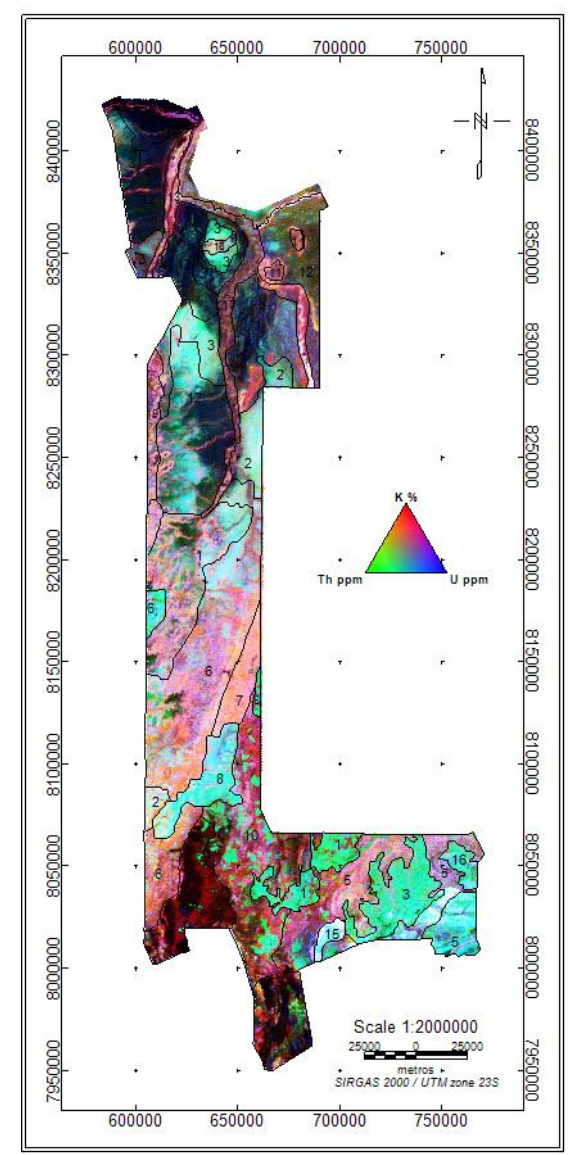

Figura 2 - Imagem da composição ternaria RGB gerada a partir dos canais de $K$, U e Th
A interpretação dos dados gamaespectrométricos foi efetuada, principalmente, através de composições ternárias do tipo RGB (Figura 2) e do tipo CMY (Figura 3). Tal análise possibilitou a definição de domínios com assinaturas gamaespectrométricas semelhantes, que foram classificadas para a elaboração do mapa litogeofísico.

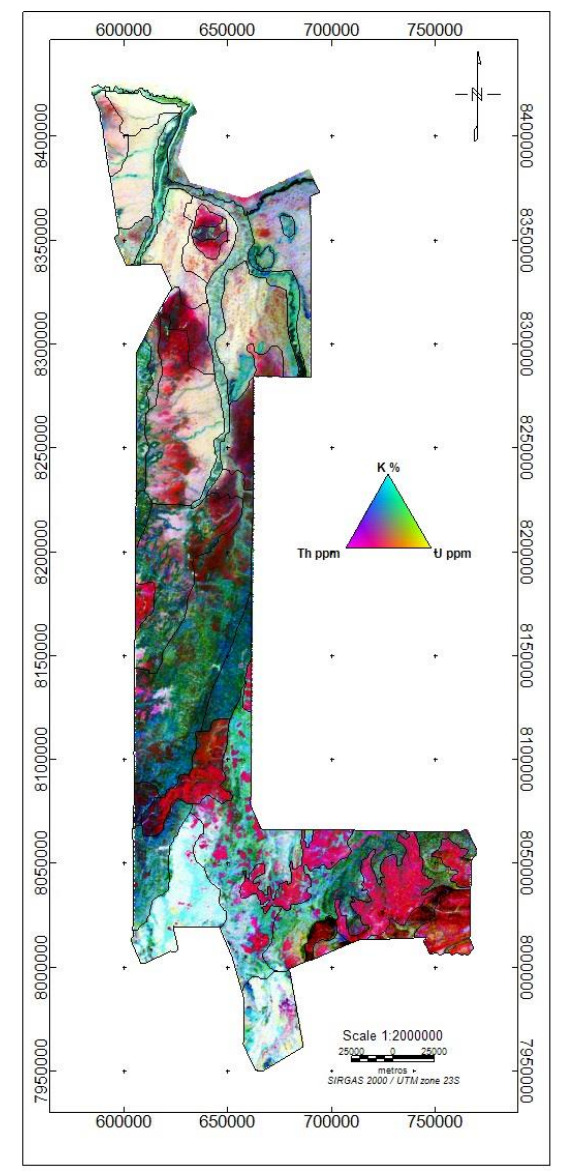

Figura 3 - Imagem da composição ternaria CMY gerada a partir dos canais de $\mathrm{K}, \mathrm{U}$ e Th.

Já na etapa de processamento dos dados magnetométricos foram produzidos produtos derivados do campo magnético anômalo (CMA) para interpretação da área. A partir do mapa do campo magnético anômalo foi gerado o mapa de amplitude do sinal analítico (ASA) para nos orientar na busca de reflexos da topografia na magnetometria (Figura 4), também foi obtido as suas três derivadas, sendo uma vertical e duas horizontais (Dx, Dy e $D z$, respectivamente). Com base nestes produtos foram traçados os lineamentos magnéticos (Figura 5) 


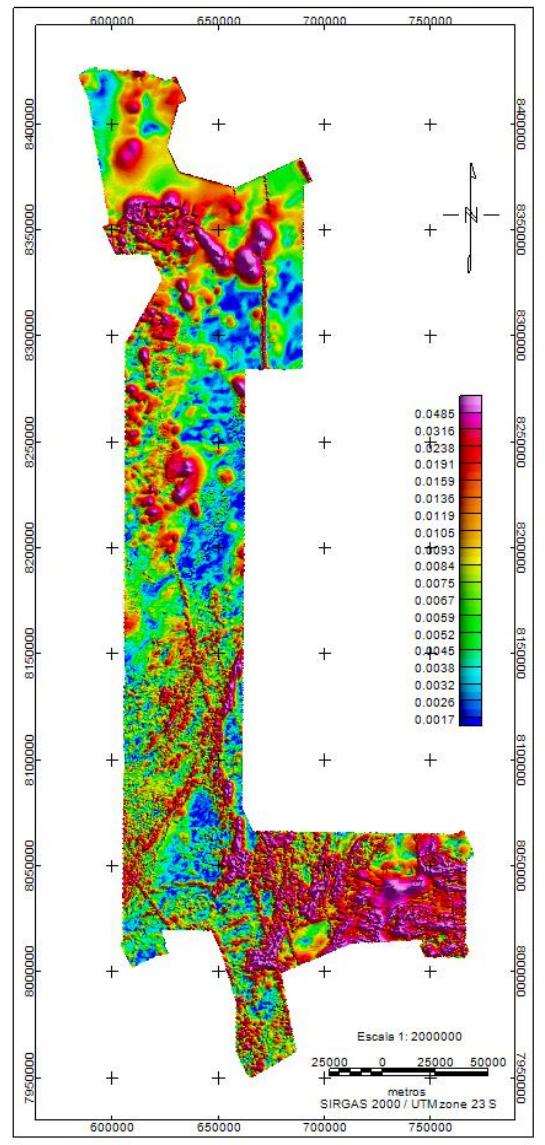

Figura 4 - Imagem da Amplitude do Sinal Analítico (ASA).

\section{Resultados e Conclusões}

As imagens de composições ternarias (RGB e CMY) foram interpretadas, com base as concentração dos teores de potássio, urânio e tório, onde foram delimitadas 22 unidades gamaespectrométricas para a região, que posterior foram relacionadas com a geologia de acordo com a geodiversidade estabelecida pela CPRM (2010), para que fosse gerado um mapa litogeofísico. (Figura 6)

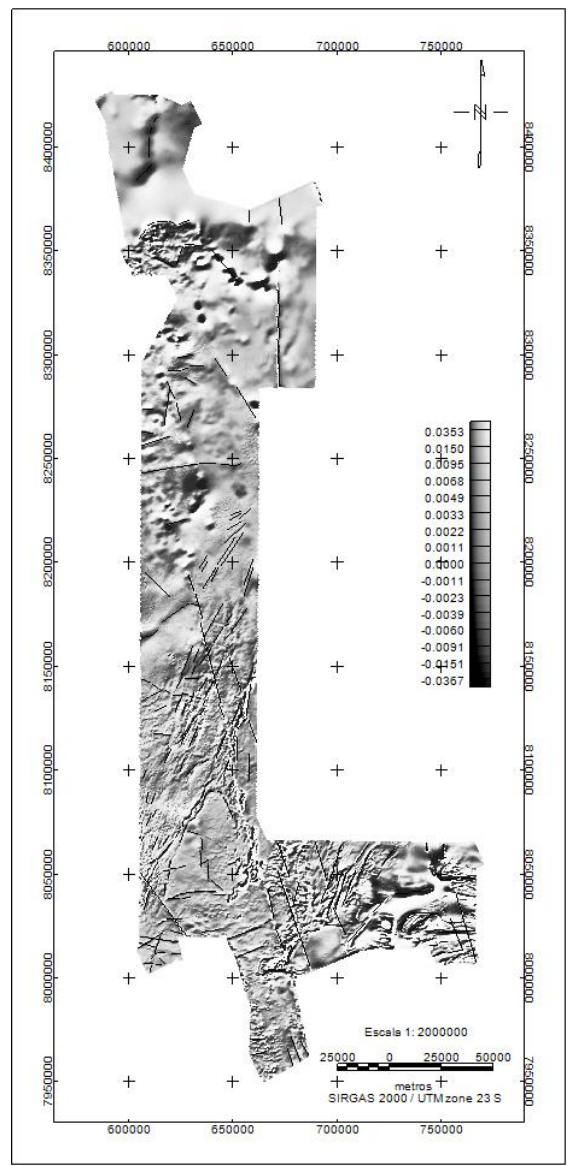

Figura 5 - Imagem da primeira derivada vertical (Dz), utilizada no mapeamento de feições lineares.

A Tabela 1 mostra as localizações e magnitudes da quatro maiores eventos que ocorreram na região central da área de estudo, no município de Montes Claros até dezembro de 2012.

\begin{tabular}{cccccc}
\hline $\begin{array}{c}\text { Data } \\
\text { AAAA-MM-DD }\end{array}$ & $\begin{array}{c}\text { Hora } \\
(\mathrm{UT})\end{array}$ & Latit. & Long. & $\begin{array}{c}\text { Prof. } \\
(\mathrm{km})\end{array}$ & Mag. \\
\hline $2012-05-19$ & $13: 41: 22.56$ & -16.6955 & -43.8828 & 1.1 & 4.0 \\
\hline $2012-09-12$ & $23: 56: 45.75$ & -16.7033 & -43.8798 & 1.2 & 2.9 \\
\hline $2012-12-19$ & $04: 54: 38.57$ & -16.6972 & -43.8783 & 1.8 & 3.6 \\
\hline $2012-12-19$ & $05: 31: 16.93$ & -16.7005 & -43.8790 & 1.5 & 3.6 \\
\hline
\end{tabular}

Tabela 1 - Maiores evento em Montes Claros, 2012. (Modificado de Assumpção, 2013) 


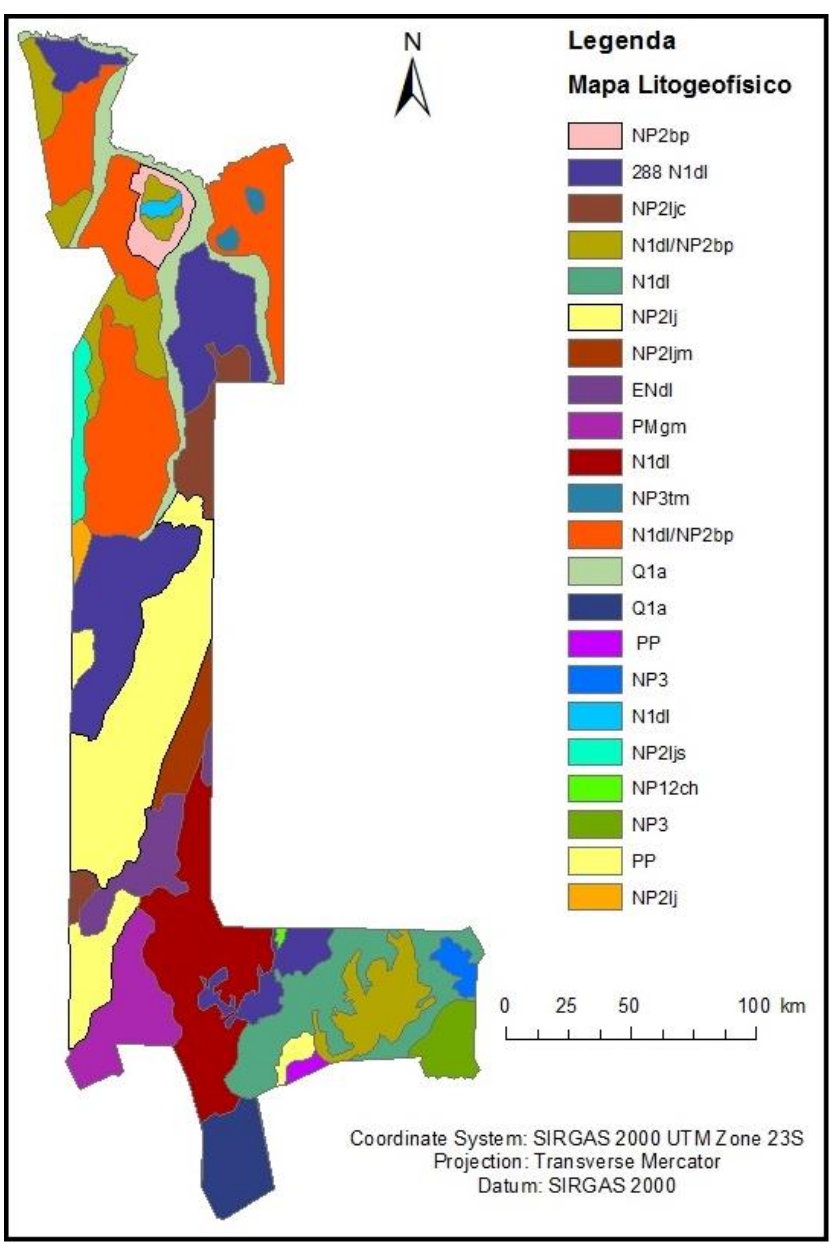

Figura 6 - Mapa litogeofísico obtido a partir de domínios gamaespectométricos.

O resultado de mecanismo focal obtido por Agurto et al, 2013, mostram a presença de esforço compressivo aproximadamente EW (Figura 7). Na região do epicentro não foi possível determinar estruturas magnéticas, mas regionalmente pode-se detectar lineamentos extensos com direção NNW-SSE e lineamentos menores NE-SW.

\section{Agradecimentos}

Agradecemos à Companhia de Desenvolvimento Econômico de Minas Gerais (CODEMIG) pela liberação dos dados do Levantamento Aerogeofísico. A primeira autora, Cláudia Teixeira, agradece ao programa $\mathrm{PIBIC/CNPq}$ pela concessão das bolsas de iniciação científica (2013 e 2014).

\section{Referências}

Agurto H., Assumpção M.; 2013 Seismotectonic characterization of the 2012 Montes Claros, Brazil, aftershock sequence. Thirteenth International Congress of the Brazilian Geophysical Society. SBGf, 2013

Alkmim, F. F.; 2004. O que faz de um cráton um cráton? O cráton do São Francisco e as revelações almeidianas ao delimitá-lo. In: Geologia do Continente Sul-Americano: Evolução da obra de Fernando Flávio Marques de Almeida, São Paulo;Editora Beca. p. 17-35.

ALMEIDA. F. F. M. 1977.0 Cráton do São Francisco. Rev. Bras. Geoc., 7(4): 349-364.

Assumpção M.; et al 2013. The 2012 Montes Claros earthquake sequence in the São Francisco craton: another evidence of inverse faulting and compressional stresses in Eastern Brazil. Thirteenth International Congress of the Brazilian Geophysical Society. SBGf, 2013

Blum M.L.B. 1999. Processamento e interpretação de dados de geofísica aérea no Brasil Central e suas aplicações à geologia e à prospecção mineral. Tese (Doutorado). IG-UnB.

DICKSON BL \& SCOTT KM. 1997. Interpretation of aerial gamma-ray surveys - adding the geochemical factors. AGSO Jour. Austr. Geol. Geoph. Austrália, 17(2): 187200.

Machado, M. F.; Silva da, S. F. (Org). Geodiversidade do Estado de minas Gerais: Programa Geologia do Brasil: Levantamento da geodiversidade de Minas Gerais. Belo Horizonte: CPRM, 2010, 133p.

CPRM. Mapa Geológico do Estado de Minas Gerais. 2003. Disponível em: http://www.cprm.gov.br/

Minty, B. R. S., 1997. Fundamentals of airborne gama-ray spectrometry. AGSO Journal of Australian Geology and Geophysics, 17(2): 39-50.

Minty, B. R. S., Luyendyk, A. P. J., 1997. Calibration and data processing for airborne gama-ray spectrometry. AGSO Journal of Australian Geology and Geophysics, 17(2): 51-62. 

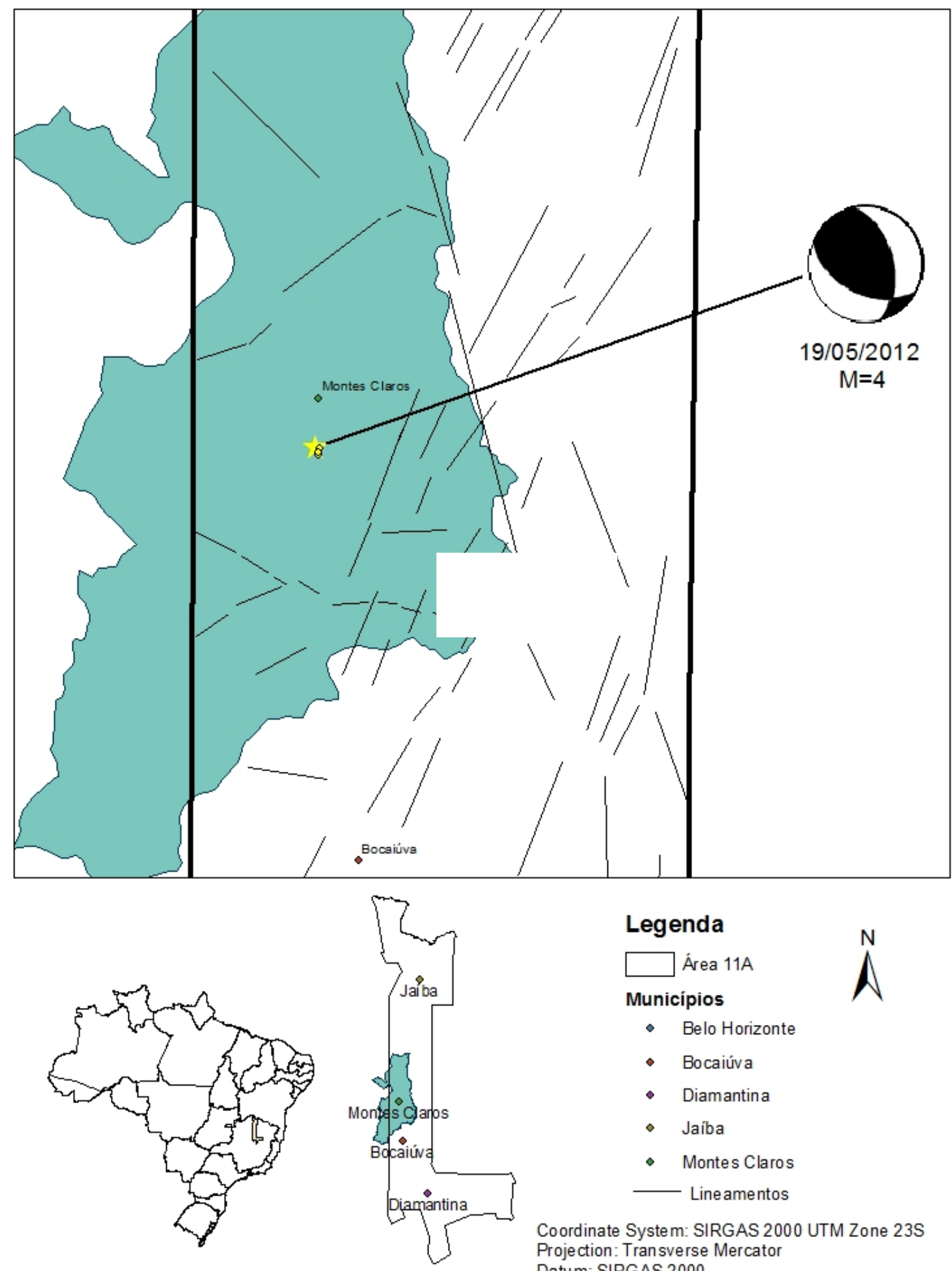

Legenda

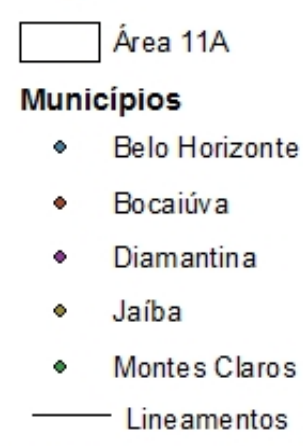

Coordinate System: SIRGAS 2000 UTM Zone 23S Projection: Transverse Mercator Datum: SIRGAS 2000

Figura 7 - A estrela amarela indica o tremor principal de magnitude 4 e ao lado seu mecanismo focal, os círculos amarelos menores se referem aos outros eventos citados na tabela 1. 